\title{
Characterizing mechanical behavior of atomically thin films: A review
}

\author{
Changhong Cao, Yu Sun, a) and Tobin Filleter ${ }^{\text {b) }}$ \\ Department of Mechanical and Industrial Engineering, University of Toronto, Ontario M5S 3G8, Canada
}

(Received 13 September 2013; accepted 8 October 2013)

\begin{abstract}
Atomically thin films, such as graphene, graphene oxide, hexagonal-boron nitride (h-BN), and molybdenum disulfide $\left(\mathrm{MoS}_{2}\right)$, have attracted intensive studies to explore their properties and potential applications as next generation materials due to their outstanding mechanical, electrical, thermal, and optical properties. The study of the mechanical behavior of this class of materials is in particular interesting as it not only physically determines the potential application fields where these materials can be utilized but also has revealed unique mechanical size effects and phenomena. Researchers have been studying the mechanical properties such as elastic modulus, strength, friction, and fracture behavior of atomically thin films for over a decade now. Here, we review recent results of the mechanical characterization and understanding of this class of materials.
\end{abstract}

\section{INTRODUCTION}

Single atomic layer two-dimensional films with thicknesses of typically less than $1 \mathrm{~nm}$ are commonly known as atomically thin films. Such a class of materials includes (but is not limited to) graphene, graphene oxide, hexagonalboron nitride (h-BN), molybdenum disulfide $\left(\mathrm{MoS}_{2}\right)$, and niobium diselenide $\left(\mathrm{NbSe}_{2}\right)$. Ever since Geim and Novoselov ${ }^{1}$ won the Nobel prize in physics for their identification and characterization of monolayer graphene $(\sim 0.333 \mathrm{~nm}$ thick $)$, studies on this class of materials have boomed. Their potential industrial applications to composite materials, ${ }^{2-4}$ lubricants, ${ }^{5,6}$ electronics, ${ }^{7-9}$ batteries, ${ }^{10-12}$ optics, ${ }^{13,14}$ as well as many more emerging fields have attracted much attention in the last decade. In addition to the well-documented electronic benefits of graphene, its mechanical behavior has become of particular interest recently with respect to the above-mentioned applications. For example, graphene has been demonstrated to be one of the stiffest and strongest materials found in nature, ${ }^{15}$ which makes it a great candidate for reinforcement in composite materials. Here, we review recent experimental results on the mechanical behavior (e.g., elastic modulus, strength, friction, and fracture) of the most studied atomically thin films which include graphene, graphene oxide, and h-BN.

\section{MATERIAL SYNTHESIS}

Graphene can be produced through the following four methods:

Address all correspondence to these authors.

a)e-mail: sun@mie.utoronto.ca

b) e-mail: filleter@mie.utoronto.ca

DOI: $10.1557 /$ jmr.2013.339

\section{A. Mechanical exfoliation}

Mechanically exfoliating graphite using scotch tape to produce graphene films was the first approach demonstrated to obtain pristine graphene [Fig. 1(a)]. ${ }^{16}$ Empirical parameters such as scotch tape adhesion characteristics and exfoliation time have been demonstrated to be important for producing monolayer or few-layers graphene. The advantages of this method are that the graphene films achieved are monocrystalline, and no complicated experimental setup is needed. However, the number of layers and the size of crystal domains are not easily controllable; in addition, the application of this method is limited due to its difficulty of application in mass production.

\section{B. Chemical modification of graphite}

Oxidizing graphite to form graphite oxide or starting with expandable graphite or graphite intercalation compounds to generate colloidal suspensions of graphite sheets, and then chemically or thermally reducing to graphene are several chemical methods of producing graphene [Fig. 1(b)]. ${ }^{19}$ Using such chemical methods, mass production and controllable synthesis of graphene become possible; however, graphene produced by this approach has a major disadvantage in that the resulting graphene films often contain a significant amount of residual oxygen and high defect density. ${ }^{19}$

\section{Epitaxial growth}

Graphitic layers can be epitaxially grown on crystal substrates such as $\mathrm{SiC}^{20}$ or metals ${ }^{17}$ to produce graphene [Fig. 1(c)]. In the case of metals, the underlying metal layer can then be etched away to produce graphene that is transferable to other substrates. Graphitic layers can also 


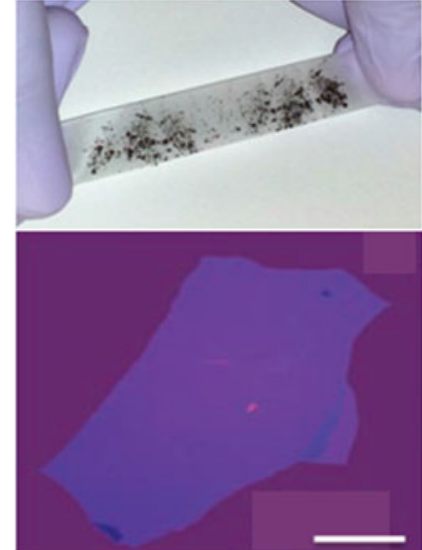

(a)

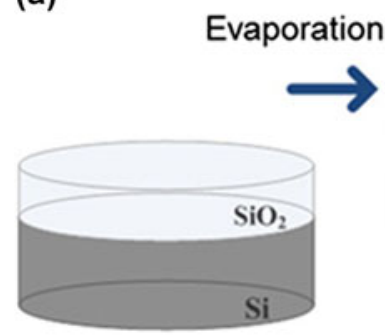

Evaporation of $\mathrm{Cu}$

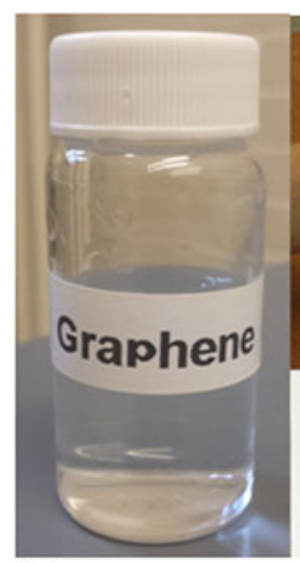

(b)

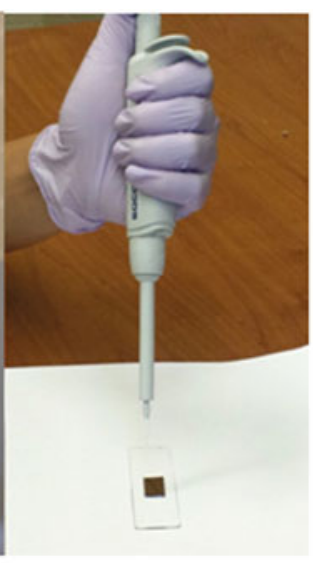

$\mathrm{H}_{2}$ annealing
(1)

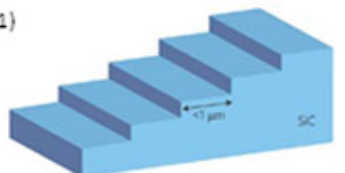

(2)

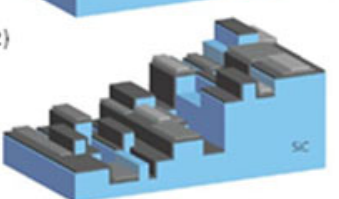

(3)

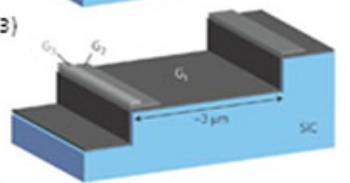

(c)

(d)

FIG. 1. Four types of graphene synthesis techniques: (a) mechanical exfoliation of graphite. Top: graphene flakes on scotch tape; bottom: 3-nm-thick graphene under an optical microscope; scale bar: $20 \mu \mathrm{m}$. Reprinted with permission from Ref. 16, Copyright 2004 Science. (b) Chemical modification of graphite. Solution-based graphene can be drop-casted on to the target substrate. (c) Epitaxial growth on SiC. (1) SiC starting surface with a staircase of flat terraces and atomic steps. (2) Schematic morphology of vacuum graphitized SiC. (3) Morphology obtained in high-pressure argon. The surface termination is predominantly monolayer graphene. Reprinted with permission from Ref. 17, Copyright 2009 Nature Publishing Group. (d) Process flow of CVD of graphene on evaporated $\mathrm{Cu}$ (111) film. Reprinted with permission from Ref. 18, Copyright 2012 American Chemical Society.

be grown on $\mathrm{SiC}$ that automatically provides an insulating substrate, which is a favorable approach for electronic devices. ${ }^{20}$ One major limitation with this method is the doping effect between the substrate and the graphitic layers during the growing process. ${ }^{21}$

\section{Chemical vapor deposition}

The chemical vapor deposition (CVD) growth approach is generally considered the most promising technique to produce industrially scalable graphene because of its flexibility [Fig. 1(d)]. ${ }^{22}$ One disadvantage is that CVD-grown graphene is typically polycrystalline which can impact its electronic and mechanical behavior. The presence of grain boundaries (GBs) in CVD graphene was initially thought to reduce the mechanical properties of graphene, but a recent study by Lee et al. ${ }^{23}$ has shown that it has nearly equivalent modulus and strength compared with that of single crystalline graphene. Furthermore, the CVD approach can be used to produce functionalized graphene such as substitutionally doped graphene ${ }^{24}$ and C-graphene. ${ }^{25} \mathrm{~A}$ summary showing the advantages and disadvantages of each method is shown in Table I.

Graphene oxide is typically synthesised by the oxidization of graphite. GO films can then be dispersed on substrate from solution in solvents such as water and ethanol with the enhancement of sonication. Three reported synthesis approaches include Brodie, Staudenmaier, or Hummers methods. ${ }^{26}$ The main difference among these methods is in the oxidizing acid used, which leads to different levels of oxidization.

Hexagonal boron nitride (h-BN) thin films can be synthesized following a similar protocol described earlier for producing graphene. Mechanical exfoliation, as illustrated above for graphene, can be used to obtain high quality but small-sized h-BN flakes. ${ }^{27}$ "Liquid phase exfoliation" 28 is a method that can obtain scalable h-BN flakes. Bulk BN crystals can be exfoliated in solvents such as isopropyl alcohol (IPA) with the assistance of sonication and centrifugation, and then dispersed onto a target substrate. CVD can produce industrially scalable h-BN thin films. However, unlike graphene synthesis, all of the abovementioned methods have yet to be shown to produce highquality monolayer h-BN, although scalable few layers h-BN can be achieved by liquid phase exfoliation and CVD. One of the reasons lies in the nature of B-N bonding (having ionic characteristics that differ from C-C covalent bonding) which results in so called "lip-lip" interactions between individual layers. ${ }^{29}$ Recently, using ammonia borane as a precursor was demonstrated ${ }^{30}$ for ambient pressure CVD growth of hboron Pt foils with controlled layers. 
TABLE I. Comparison of four major graphene synthesis methods.

\begin{tabular}{|c|c|c|}
\hline Graphene synthesis & Advantages & Disadvantages \\
\hline \multirow[t]{2}{*}{ Mechanical exfoliation } & Pristine graphene $e^{16}$ & Uncontrollable domain size and layer number \\
\hline & Simple synthesis procedure & Cannot be industrially scalable \\
\hline \multirow{2}{*}{$\begin{array}{l}\text { Chemical modification } \\
\text { of graphite }\end{array}$} & Controllable synthesis & Oxygen residue \\
\hline & Can be mass produced & High defect density ${ }^{19}$ \\
\hline Epitaxial growth & $\begin{array}{l}\text { Controllable synthesis and mass producible } \\
\text { Favorable approach for electronics }{ }^{20}\end{array}$ & Doping effect to the graphitic layers during synthesis ${ }^{21}$ \\
\hline CVD & $\begin{array}{l}\text { Controllable and flexible synthesis } \\
\text { Industrially scalable } \\
\text { Easy to be further functionalized }{ }^{24,25}\end{array}$ & Modulus and strength of the material can weaken if handled improperly ${ }^{23}$ \\
\hline
\end{tabular}

\section{ELASTIC MODULUS AND STRENGTH}

Elastic modulus and strength, as two important mechanical properties of materials, of atomically thin films have drawn intensive attention. Challenges in characterizing mechanical properties of atomically thin films include well-defined sample geometries, stress concentration at boundaries, measuring low forces, and developing mechanics models for determining stress. To overcome this limitation, Lee et al. ${ }^{15}$ performed atomic force microscopy (AFM) measurements on suspended thin films on Si wafers with circular holes (diameter $\sim 2 \mu \mathrm{m}$ ) to determine elastic modulus and strength [Fig. 2(a) ]. The method is similar to previously developed methods for investigating the mechanical behavior of thin metal films, where a nanoindenter was used instead of an AFM for force and deflection measurements. ${ }^{33}$ The AFM deflection technique eliminates both the boundary condition problem and the substrate effect, which enables nanoindentation to be an ideal way to measuring modulus and strength for this class of materials. In addition, graphitic ultrathin films are found to adhere to Si strongly; therefore, no observable slippage occurs during indentation or scanning, presumably due to van der Waals attractions between the film and the substrate. ${ }^{15}$ This technique has been applied to characterize various 2D ultrathin films (graphene, graphene oxide, and boron nitride).

Graphene was the first atomically thin film that was characterized using the above mentioned technique. Lee et al. ${ }^{15}$ mechanically exfoliated graphite onto a micromachined Si substrate to form monolayer suspended pristine graphene with well-defined circular boundary conditions [Fig. 2(a)]. Indentation tests were performed at the center of the suspended membrane for elastic modulus and strength measurements. A nonlinear elastic response was governed by the third order stress strain relationship:

$$
\sigma=E \varepsilon+D \varepsilon^{2}
$$

where $\sigma$ is the symmetric 2 nd Piola-Kirchhoff stress, $\varepsilon$ is the uniaxial Lagrangian strain, $E$ is the Young's modulus, and $D$ is the 3rd order elastic modulus. Based on statistical analysis, $E$ was determined to be $1.0 \pm 0.1 \mathrm{TPa}$ and
$D$ was $-2.0 \pm 0.4 \mathrm{TPa}$ assuming an effective graphene thickness of $0.335 \mathrm{~nm}$. As mentioned above, graphene produced by mechanical exfoliation is proven to be single crystalline with a low defect density, based on which the measured strength can be viewed as the intrinsic strength of monolayer graphene. Assuming the indenter has a spherical shape and ignoring the nonlinear elasticity, the maximum stress under this configuration can be modeled as

$$
\sigma_{m}^{2 D}=\left(\frac{F E^{2 D}}{4 \pi R}\right)^{\frac{1}{2}}
$$

where $\sigma_{m}^{2 D}$ is the maximum stress at the center of the film, $F$ is the maximum force, and $R$ is the radius of the indenter. Combining nonlinear elasticity, the calculated intrinsic strength was $130 \pm 10 \mathrm{GPa}$ at $25 \%$ strain. This work is the first comprehensive measurement of elastic properties of monolayer graphene, which set the benchmark for mechanical characterization of ultrathin films.

Mechanically exfoliated graphene has limited applications due to manufacturing limitations. However, graphene produced by the CVD method is a promising approach to produce industrially scalable materials. Earlier measurements of CVD graphene showed that due to the existence of grain boundaries (GBs), the elastic stiffness degrades to $\sim 165 \mathrm{GPa}^{34,35}$ Lee et al. ${ }^{23}$ recently provided a possible explanation as to why the previous practices can degrade the elastic stiffness and proposed improvement to keep graphene intact during fabrication. They claimed that the problem was the creation of ripples during the graphene transfer process. It is known that CVD grown graphene is typically synthesized on metals (e.g., copper). During the dry transfer process, Poly (methyl methacrylate) (PMMA) is coated on top of graphene as a handle layer and then the copper substrate is etched away. After stamping graphene onto the target substrate, the PMMA layer is also removed. Lee et al. found the etching solvents and the removing step created the ripples. When the copper etchant is changed to ammonium persulfate $\left[\left(\mathrm{NH}_{4}\right)_{2} \mathrm{~S}_{2} \mathrm{O}_{8}\right]$ instead of ferric chloride $\left(\mathrm{FeCl}_{3}\right)$ and the air-baking step during PMMA removal is eliminated, ripples are largely reduced. Following the same 


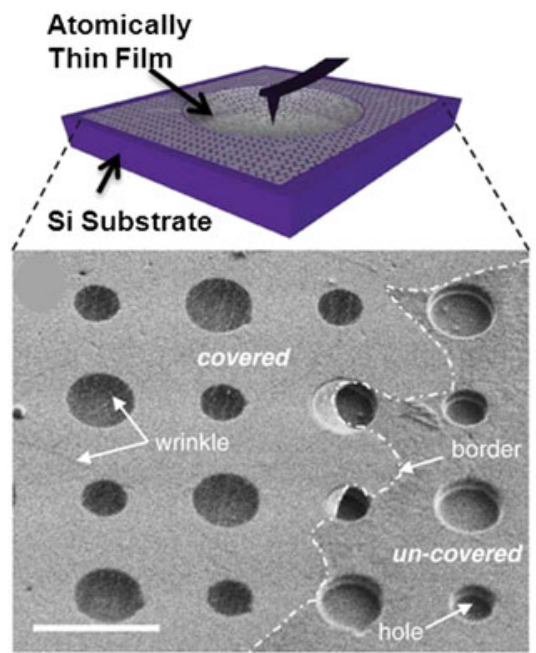

(a)

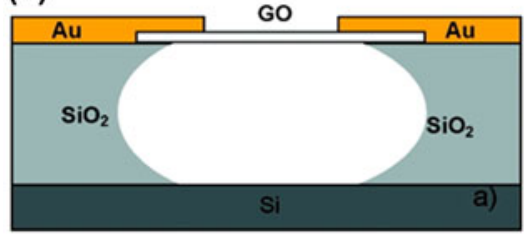

(c)

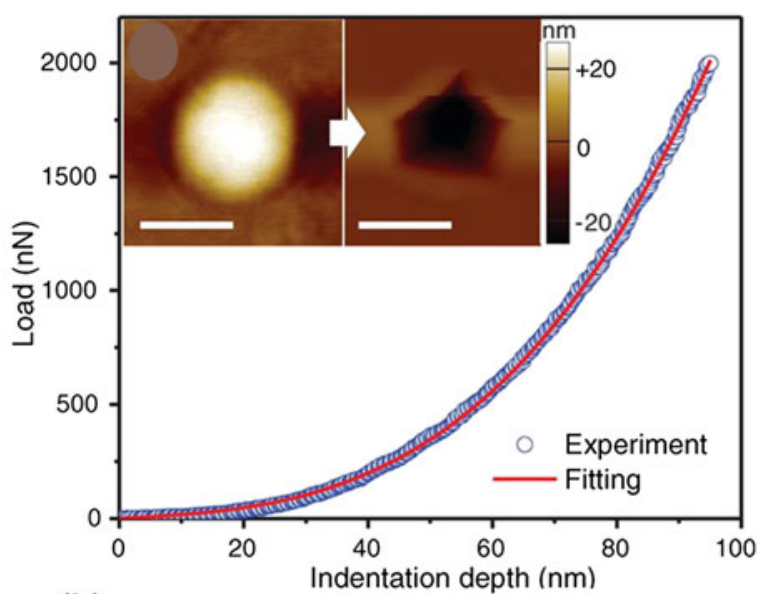

(b)

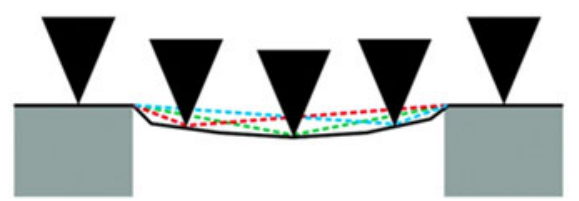

(d)

FIG. 2. (a) Graphene membrane suspended over the Si substrate with circular holes. Reprinted with permission from Ref. 23 , Copyright 2013 Science. (b) Force-displacement curve of CVD graphene film in AFM nanoindentation. Inset: the AFM topology images of the suspended graphene film before and after fracture. Reprinted with permission from Ref. 23, Copyright 2013 Science. (c) Graphene oxide film suspended over trenches. Reprinted with permission from Ref. 31, Copyright 2008 American Chemical Society. (d) Graphene oxide film suspended over holey carbon substrate. Reprinted with permission from Ref. 32, Copyright 2010 American Chemical Society.

testing protocol as for pristine graphene, Lee et al. showed that the elastic moduli for both large graphene (LG) and small graphene (SG) are approximately $1 \mathrm{TPa}$ [Fig. 2(b)]. They concluded that there are no statistical differences between the two types. Under the same testing configuration, the breaking strength of SG, LG, and pristine graphene was measured as well. The results demonstrate that LG and pristine graphene have an equivalent strength of $118 \mathrm{GPa}$, and SG has slightly lower strength of $98.5 \mathrm{Gpa}$, which was attributed to randomly distributed defects and GBs.

The first measurements of the mechanical properties of graphene oxide films were conducted on GO reduced graphene by Gómez-Navarro et al. $^{31}$ Such materials do not exhibit a pristine graphene structure and have been shown to still contain many $\mathrm{O}$ groups following reduction giving them a hybrid graphene-GO structure. ${ }^{36}$ GómezNavarro et al. adopted a testing configuration of suspending reduced $\mathrm{GO}$ over a $\mathrm{Si} / \mathrm{SiO}_{2}$ trench [Fig. 2(c)]. Indentation tests were performed at the center of the film. Elastic modulus of the film was extracted according to

$$
E=\frac{32(l / t)^{3} K_{\mathrm{eff}}}{\omega}-17 T l^{2} / 32 \omega t^{3},
$$

where $E$ is the elastic modulus, $K_{\text {eff }}$ is the stiffness which can be obtained through the force distance curve from indentation, $t, \omega$, and $l$ are the thickness, width, and length of the suspended film, $T$ is the pretension in the film. This model is based on the double-clamped beam theory. For small indentation depth (pure bending regime) and point load at the center, force constants depend both on the properties and geometry of the material, which is different than Lee's model [Eq. (1)]. The results showed that the elastic modulus was $0.25 \pm 0.15 \mathrm{TPa}$.

Suk et al. ${ }^{32}$ directly measured the elastic modulus of monolayer graphene oxide by a similar experimental setup as Lee et al. ${ }^{15}$ Instead of using a Si-based substrate to suspend the film, they used a carbon film-based TEM grid with circular holes, which, while providing well-defined boundary conditions and good adhesion between the film and the substrate, has a limitation in that the supporting membrane is very compliant in the normal direction. Rather than doing indentation on the film, they used a technique of solely scanning the film surface using contact mode AFM [Fig. 2(d)]. By applying different loads while scanning the surface, deflection of the film varies. Coupling with finite element analysis, the reduced modulus of monolayer graphene oxide was measured to be $207.6 \pm 23.4 \mathrm{GPa}$. Assuming bending stiffness to be zero, the Young's modulus was also determined to be $156.5 \pm 23.9 \mathrm{GPa}$. These results are in agreement with those reported by Gómez-Navarro et al. ${ }^{31}$ The advantages of this method are 


\section{Graphene}

(1)

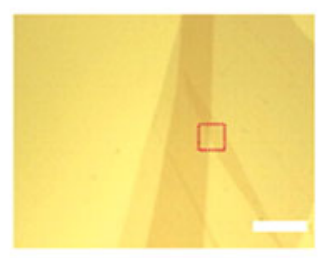

(2)

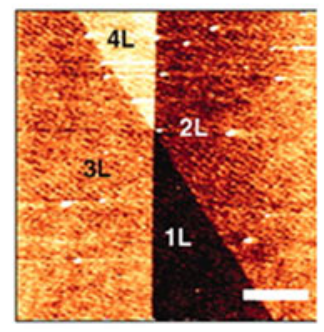

(3)

(4)
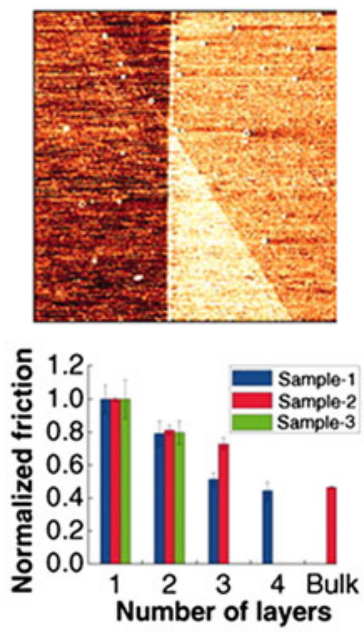

(a)

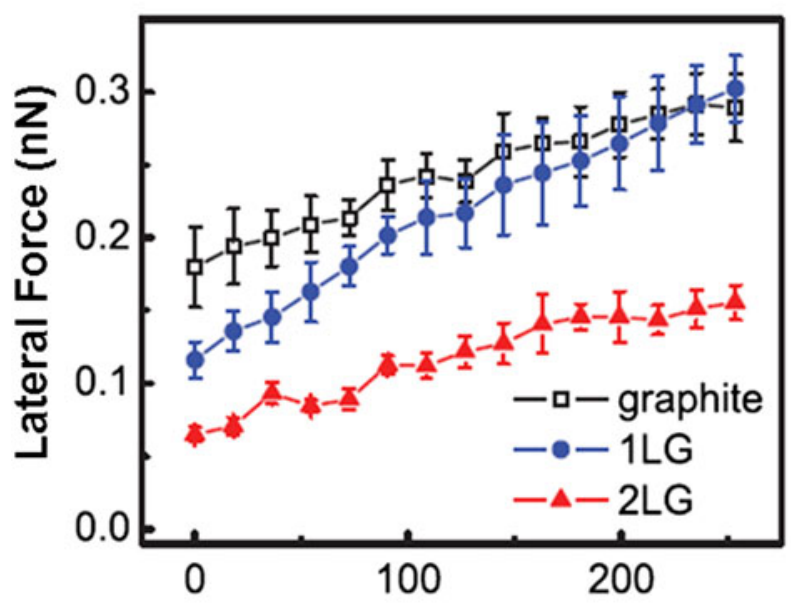

(b)

\section{Normal Force $(\mathrm{nN})$}

FIG. 3. (a) Optical and AFM images of atomically thin sheets of graphene and h-BN on silicon dioxide: (1) bright-field optical microscope images of thin sample flakes. The red dotted squares represent subsequent AFM scan areas. Scale bars, $10 \mu \mathrm{m}$. (2 and 3) Topographic and friction (forward scan) images measured simultaneously by AFM from the indicated areas. 1L, 2L, 3L, etc. indicate sheets with thicknesses of one, two, three, etc. atomic layers. BL ("bulk-like") denotes an area with a very that it avoids large load and sample damage; the problem of indentation induced jumping or snapping of AFM tips ${ }^{37}$ is minimized; and pretension in the film can also be considered and calculated. Most importantly, the strength of the film cannot be measured on a soft substrate. To our knowledge, the strength of monolayer graphene oxide has not yet been reported, although it is a critical parameter considering the use of graphene oxide in macroscopic materials. $^{38}$

Boron nitride few-layer films produced by CVD were mechanically tested by Song et al. ${ }^{39}$ h-BN films (1- to 2-nm thick, 3-5 layers) were tautly covered on a Si wafer with $1 \mu \mathrm{m}$ holes, forming a similar experimental setup to the one reported by Lee et al. ${ }^{15}$ Indentation tests were performed to extract the Young's modulus and strength of the films. The theoretical model used for analyzing experimental data was also the same as that used by Lee et al. ${ }^{15}$ The reported results were $223 \pm 16 \mathrm{GPa}$ and $8.8 \pm 1.2 \mathrm{GPa}$ for elastic stiffness and breaking strength, which are comparable to pristine graphene $(\sim 1 \mathrm{TPa}$ and $130 \mathrm{GPa}$ ). They also concluded that with the increase in layers, both Young's modulus and strength of the film increase. Elastic modulus and strength for monolayer and pristine h-BN thus far have not been reported.

Elastic modulus of other 2D atomically thin films were also measured based on the "suspension" technique. For instance, Castellanos-Gomez et al. ${ }^{40}$ measured elastic modulus of few-layer $\mathrm{MoS}_{2}(\sim 5$ layers $)$ to be $0.33 \pm 0.07 \mathrm{GPa}$ (strength was not reported). From the above-reported elastic modulus of these 2D ultrathin films, one can see that this is an extremely strong class of materials. Unfortunately, the strength of graphene oxide has not yet been reported. Fabrication techniques of this class of materials vary, creating defect density differences among even the same type of material. Elastic modulus and strength produced/ transferred by different approaches may vary correspondingly. Systematic studies of elastic properties of films synthesized by different methods, especially for GO, h-BN, and $\mathrm{MoS}_{2}$, still need to be performed.

\section{FRICTIONAL PROPERTIES}

The frictional properties of graphene produced by epitaxial growth on a $\mathrm{SiC}$ substrate were characterized

thick flake, and $\mathrm{S}$ represents an area with bare $\mathrm{SiO}_{2}$ substrate. Scale bars, $1 \mu \mathrm{m}$. (4) Friction on areas with different layer thicknesses. For each sample, friction is normalized to the value obtained for the thinnest layer. Error bars represent the standard deviation of the friction signals of each area. In each chart, the same color represents data from the same sample. Reprinted with permission from Ref. 42, Copyright 2010 Science. (b) Normal versus lateral force measurement on single layer (1LG), bilayer (2LG), and graphite. Reprinted with permission from Ref. 41, Copyright 2009 Physical Review Letters. 

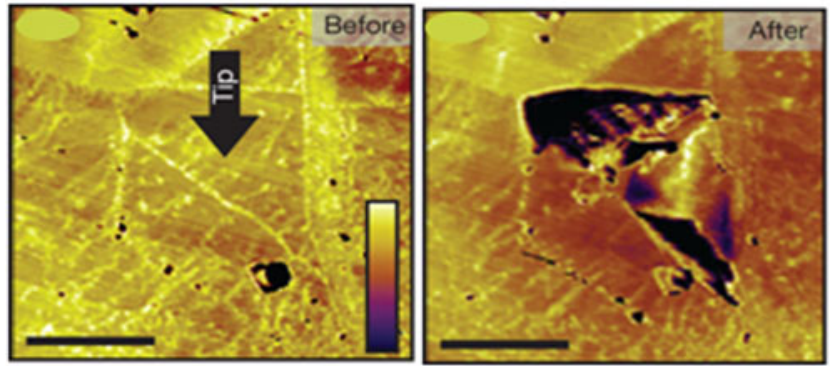

(a1)

(a2)

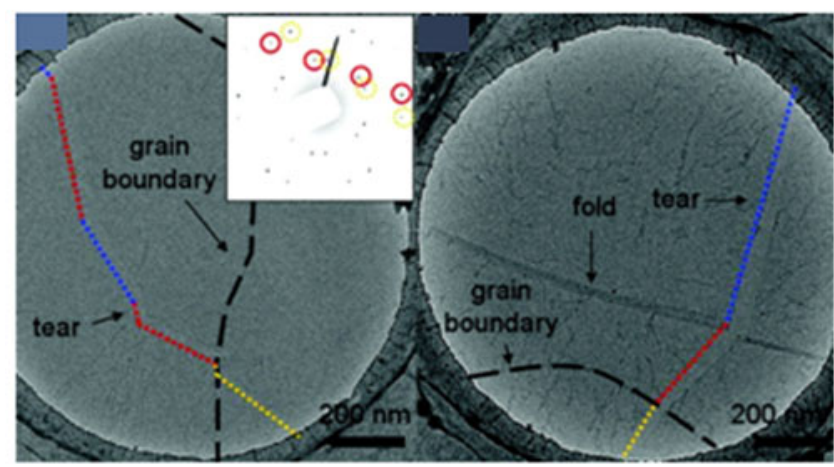

(b1)

(b2)
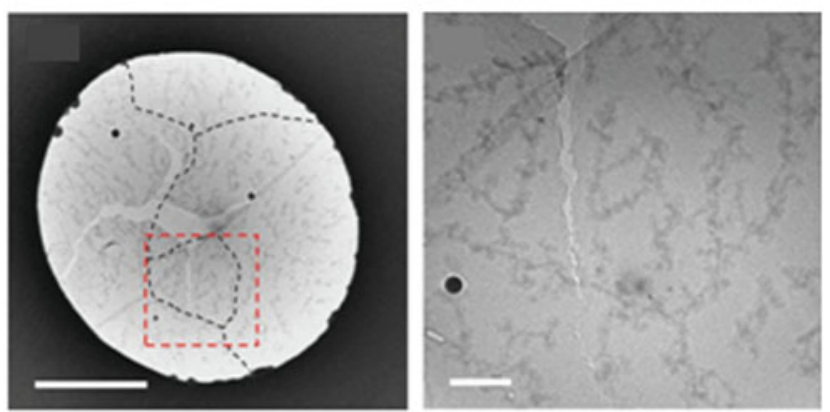

(c1)

(c2)

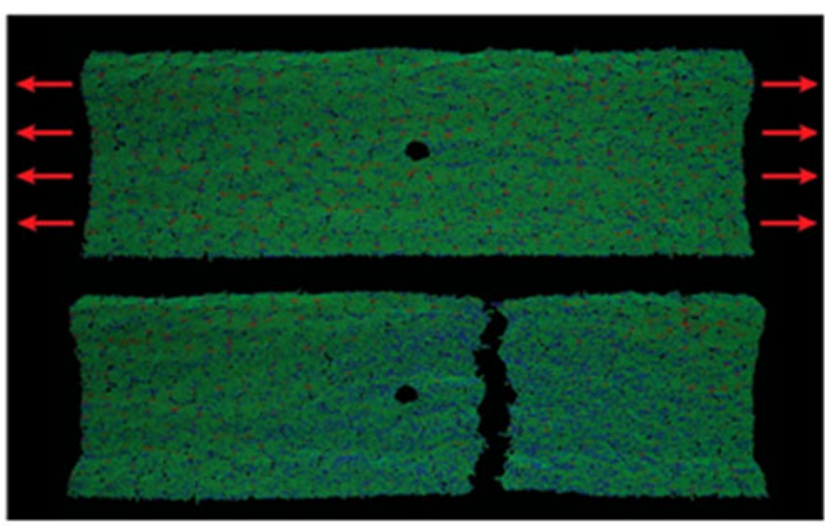

(d1)

(d2)

FIG. 4. (a) AFM phase images of a graphene grain before and after an indentation measurement. (a1) Indentation takes place at the center of this grain as shown by the arrow. (a2) The region is torn along GBs after indentation. Reprinted with permission from Ref. 34, Copyright 2011 Nature Publishing Group. (b) Graphene tears crossing GBs. (b1) TEM image of graphene tear crossing, not following, a GB in graphene. The GBs are identified by the high absorbent concentration along the GB by Filleter et al. ${ }^{41}$ The results showed that friction on single-layer graphene is higher than that of bilayer graphene. Similar results were reported by Lee et al. ${ }^{42}$ demonstrating that the friction of the graphene film has an inverse proportional relationship with layer thickness. Single-layer graphene shows the highest friction, whereas samples with a thickness of approximately four layers exhibit the lowest friction and approach levels of bulk graphite [Fig. 3(a), left]. However, for bulk graphite samples, there was some discrepancy between these studies. Filleter et al. observed that friction on graphite is higher than single-layer graphene due to higher adhesion, which can be seen from the normal versus lateral force curve comparison between 1LG, 2LG, and graphite [Fig. 3(b)]. Bilayer graphene and graphite have the same slope suggesting a similar friction coefficient, but with the same normal load, graphite shows a higher offset lateral force indicating higher adhesion between graphene and the tip. Lee et al. suggested that graphite has higher bending stiffness compared with few layer graphene so that the lateral motion on graphite (more than 5 layers) is easier than on few-layer graphene. For single and several-layer graphene, Lee et al. also showed that substrate (loosely adhere or suspended), scan size, and scan rate differences would not affect the trend. However, if the graphene film adheres strongly to the underlying substrate, the trend would be suppressed. In other words, friction on few-layer graphene is almost identical to graphite if they are well adhered to the substrate. This insight provides potential opportunities to control tribological effects during applications. Lee et al. and Filleter et al. together comprehensively interpreted the frictional properties of mechanically exfoliated and epitaxially grown graphene. However, frictional properties of graphene produced by other methods such as CVD and chemically reduced graphene are yet to be studied.

h-BN fabricated by mechanical exfoliation was the only type of h-BN material whose frictional property has been studied. The relationship between friction and layer

together with electron diffraction. The inset of the diffraction pattern around the GB showing two sets of hexagonal patterns from two adjacent tilt grains, where the hexagonal pattern marked with red and dashed yellow circles corresponds to the grain in the left and right sides, respectively. The blue dotted lines represent tear lines in the zigzag direction. The red and yellow dotted lines represent tear lines in the armchair direction. (b2) Another TEM image of a graphene tear crossing a GB and fold in graphene. The blue dotted line represents a line in the zigzag direction. The red and yellow dotted lines represent the armchair direction. Reprinted with permission from Ref. 45, Copyright 2012 American Chemical Society. (c) (c1) BF-TEM image after indentation. The black dashed lines indicate GBs. (c2) Enlarged BFTEM image of the red dashed area of (c1). Reprinted with permission from Ref. 23, Copyright 2013 Science. (d) Simulation of crack propagation on polycrystalline graphene with existing flaws. Reprinted with permission from Ref. 46, Copyright 2012 American Chemical Society. 
thickness for h-BN has a similar trend to graphene, proposed by Lee et al. ${ }^{42}$ As the number of layers increases, the friction reduces accordingly if the film is loosely adhered to or suspended on the underlying substrate [Fig. 3(a), right]. Because of the difficulty of producing single- or few-layer h-BN films, the amount of experimental data was limited for drawing conclusions. Additionally, whether h-BN films produced by other methods result in differences in frictional properties is not known yet.

Graphene oxide of single or few layers has not been systematically studied in terms of frictional properties, although $\mathrm{Ou}$ et al. ${ }^{43}$ reported that reduced graphene oxide sheets can be used to reduce wear life under small loads. Tribological behavior of few-layer graphene oxide was only preliminarily reported qualitatively. ${ }^{44}$ Furthermore, Wei et al. $^{44}$ found that thermal reduction can lower the friction of graphene oxide by replacing it with graphene. Graphene oxide has a wide range of applications; hence, its frictional properties deserve further studies, in particular due to the capabilities to functionalize its surfaces.

\section{FRACTURE BEHAVIOR}

Fracture behavior is another fundamental material property, which is critical in understanding the failure of materials. Even a single crack can cause the entire failure of a material. For 2D ultrathin films, stress concentrations can lead to crack formation and growth. Graphene is the only atomically thin film that has been characterized in terms of fracture behavior. The fracture behavior of graphene oxide, h-BN, and $\mathrm{MoS}_{2}$ has not been reported to date.

Fracture behavior was experimentally characterized on CVD-produced graphene due to its polycrystalline nature. Huang et al. ${ }^{34}$ utilized a dark-field transmission electron microscope to obtain high-resolution images of grains and GBs of graphene [Fig. 4(a)]. By doing so, they mapped the location and the tilt orientation of grains and further found that different grains are stitched together mainly by pentagon-heptagon pairs. Indentation was performed to test the strength of the membrane, following the protocol of suspension of a film over a holey substrate. ${ }^{15}$ The key findings are that the breaking load is approximately 1.7 orders of magnitude lower than pristine graphene, and cracks propagate along GBs. A similar finding was observed by Kim et al. ${ }^{45}$ that electron excitation-initiated cracks prefer to propagate either along armchair or zigzag directions, indicating that GBs are weaker regions [Fig. 4(b)]. However, Lee et al. ${ }^{23}$ recently performed indentation directly on GBs, showing that an intergranular crack was formed under the tip. This confirmed GBs are somewhat weaker than graphene, but cracks later propagate into adjoining grains, and the torn edges of the transgranular cracks have an irregular sawtooth shape [Fig. 4(c)]. Also, their measured strength of GBs is much higher than previous studies because of their better sample transfer techniques.

Analyzing the above three studies, ${ }^{23,34,45}$ one can notice that as the sample preparation and transfer techniques become more mature, the weakening effect on strength caused by the presence of GBs becomes less pronounced. However, due to the disagreement of experimental observance of crack propagation, further studies are needed to explore the physics behind how and why cracks form and propagate in this class of ultrathin materials. Toward this end, through molecular dynamic simulations, Zhang et al. ${ }^{46}$ showed that polycrystalline graphene exhibits "flaw tolerance." As illustrated in Fig. 4(d), the fracture behavior of polycrystalline graphene can become insensitive to a preexisting flaw (hole or notch) below a critical length scale, suggesting that there is no stress concentration near the flaw site. Experimental verification of such an effect is yet to be conducted to confirm the failure and fracture mechanisms of graphene.

TABLE 2. Summary of state-of-the-art results.

\begin{tabular}{|c|c|c|c|c|c|c|c|c|}
\hline & \multicolumn{5}{|c|}{ Graphene } & \multirow{3}{*}{$\begin{array}{c}\text { GO } \\
\text { Chemical } \\
\text { method }\end{array}$} & \multicolumn{2}{|l|}{ h-BN } \\
\hline & \multirow{2}{*}{$\begin{array}{l}\text { Mechanical } \\
\text { exfoliation }\end{array}$} & \multirow{2}{*}{$\begin{array}{l}\text { Chemical } \\
\text { method }\end{array}$} & \multirow{2}{*}{$\begin{array}{l}\text { Epitaxial } \\
\text { growth }\end{array}$} & \multicolumn{2}{|c|}{ CVD } & & \multirow{2}{*}{$\begin{array}{l}\text { Mechanical Liquid phase } \\
\text { exfoliation exfoliation }\end{array}$} & \multirow[b]{2}{*}{ CVD } \\
\hline & & & & LG & SG & & & \\
\hline Elastic Modulus (GPa) & $\sim 1000^{15}$ & $\cdots$ & $\cdots$ & \multicolumn{2}{|c|}{$\sim 165^{34,35}$} & $\begin{array}{c}250 \pm 150^{31} \\
156.5 \pm 23.9^{32}\end{array}$ & \multicolumn{2}{|r|}{$223 \pm 16^{39}$} \\
\hline Strength (GPa) & $130 \pm 10^{15}$ & $\ldots$ & $\ldots$ & $103^{23}$ & $98.5^{23}$ & $200-30$ & $\ldots \quad \ldots$ & $8.8 \pm 1.2^{39}$ \\
\hline Friction & $\begin{array}{r}\text { Mechanically e } \\
\text { - The thinne } \\
\text { Epitaxially gro } \\
\text { - Double lay } \\
\text { - Friction co }\end{array}$ & $\begin{array}{l}\text { iated: } \\
\text { higher } \\
42 \\
\text { as lower fri } \\
\text { ient: single }\end{array}$ & $\begin{array}{l}\text { than singl } \\
.0048) \text { and }\end{array}$ & $\operatorname{er}^{41}$ & )$^{41}$ layers & $\cdots$ & $\begin{array}{l}\text { Mechanically exfol } \\
\text { - The thinner the hi }\end{array}$ & $\begin{array}{l}\text { liated: } \\
\text { igher }^{42}\end{array}$ \\
\hline Fracture & $\begin{array}{l}\text { CVD: } \\
\text { - Fracture cr } \\
\text { - GBs are w } \\
\text { - Fracture cr }\end{array}$ & $\begin{array}{l}\text { move both } \\
\text { than pristi } \\
\text { move pref }\end{array}$ & $\begin{array}{l}\text { ng and into } \\
\text { raphene }{ }^{34} \\
\text { ly along arn }\end{array}$ & $s^{23}$ & ections ${ }^{45}$ & $\ldots$ & $\ldots$ & $\cdots$ \\
\hline
\end{tabular}




\section{SUMMARY}

Graphene has been the most extensively studied of the atomically thin film class of materials that have been reviewed here. As summarized in Table II, a wide range of mechanical properties (elastic modulus, strength, friction, and fracture) of graphene has been reported, though property comparisons among graphene produced by different techniques are not complete.

Graphene oxide's strength, friction, and fracture behavior still await further investigation. This is due in part to the challenges involved in fabricating large, flat, and uniform samples similar to those that can be produced by exfoliating graphene. Fracture behavior of h-BN is not known, and comparisons of the other three mechanical properties of h-BN produced by different methods also are yet to be studied. Deeper exploration including additional smallscale mechanical characterization of atomically thin films is needed. This will require advances in instrumentation. For example, the dislocation and fracture behavior of films is an important fundamental mechanical property of materials, which if well understood, can be used to predict failure mechanisms. To date, its study in such small systems has been limited by insufficient methodologies and tools.

To achieve this goal, fast and high-resolution microscopy techniques coupled with specially designed devices that can handle the materials of this scale are required. Dynamic $\mathrm{TEM}^{47}$ is an emerging technique which can already provide temporal resolution of structural changes observed by TEM in materials on the order of approximately nanoseconds. Further advances will be required to temporally resolve dislocation propagation. Microelectromechanical system (MEMS) devices can also be designed and applied to perform a number of mechanical tests on atomically thin films in situ electron microscopes; however, the reported MEMS devices for studying 1D nanostructures ${ }^{48,49}$ such as nanotubes and nanowires ${ }^{50}$ have yet to be extended to 2D films.

Further understanding of the mechanical properties of atomically thin films can greatly impact their applications. For example, industrially scalable atomically thin film-based materials are promising to be applied to transportation and electronic applications due to their superior mechanical and electrical properties. ${ }^{2}$ Large-scale production methods of atomically thin films such as CVD growth and chemical exfoliation have the potential to create next generation industrially relevant materials, such as composites and stretchable transparent electrodes/electronic devices based on graphene and h-BN. ${ }^{11,51}$

A number of emerging applications of this class of materials depend critically on the fundamental understanding of their properties to tailor their properties to match specific needs. For instance, tribological properties are important to characterize for lubrication and wear applications in MEMS and steel mechanical compo- nents. ${ }^{41,42,52}$ In battery technologies, thin films are used to enhance the performance of anodes and cathodes (e.g., $\mathrm{Li}$ ion batteries), based on the inclusion of atomically controlled film layers. ${ }^{12}$ Mechanical characterization will play a particularly important role in solid-state batteries that incorporate multiple thin films and interfaces of different material compositions. As more intensive research efforts are spent on making functionalized atomically thin films, extensive mechanical property characterization will become even more important, in particular due to the size effects that emerge for such confined systems as compared to their bulk counterparts.

\section{REFERENCES}

1. A.K. Geim and K.S. Novoselov: The rise of graphene. Nat. Mater. 6, 183-191 (2007).

2. S. Stankovich, D.A. Dikin, G.H.B. Dommett, K.M. Kohlhaas, E.J. Zimney, E.A. Stach, R.D. Piner, S.T. Nguyen, and R.S. Ruoff: Graphene-based composite materials. Nature 442, 282-286 (2006).

3. S. Watcharotone, D.A. Diking, S. Stankovich, R. Pinery, I. Jung, G.H.B. Dommett, G. Evmenenko, S-E. Wu, S-F. Chen, C-P. Liu, S.T. Nguyen, and R.S. Ruoff: Graphene-silica composite thin films as transparent conductors. Nano Lett. 7, 1888-1892 (2007).

4. G. Eda and M. Chhowalla: Graphene-based composite thin films for electronics. Nano Lett. 9, 814-818 (2009).

5. E.J. Sandoz-Rosado, O.A. Tertuliano, and E.J. Terrell: An atomistic study of the abrasive wear and failure of graphene sheets when used as a solid lubricant and a comparison to diamond-like-carbon coatings. Carbon 50, 4078-4084 (2012).

6. K-S. Kim, H-J. Lee, C. Lee, S-K. Lee, H. Jang, J-H. Ahn, J-H. Kim, and H-J. Lee: Chemical vapor deposition-grown graphene: The thinnest solid lubricant. ACS Nano 5, 5107-5114 (2011).

7. P. Sungjin, A. Jinho, S. Ji Won, and R.S. Ruoff: Graphene-based actuators. Small 6, 210-212 (2010).

8. C.R. Dean, A.F. Young, I. Meric, C. Lee, L. Wang, S. Sorgenfrei, K. Watanabe, T. Taniguchi, P. Kim, K.L. Shepard, and J. Hone: Boron nitride substrates for high-quality graphene electronics. Nat. Nanotechnol. 5, 722-726 (2010).

9. O. Lopez-Sanchez, D. Lembke, M. Kayci, A. Radenovic, and A. Kis: Ultrasensitive photodetectors based on monolayer $\mathrm{MoS}_{2}$ Nat. Nanotechnol. 8, 497-501 (2013).

10. N. Li, Z.P. Chen, W.C. Ren, F. Li, and H.M. Cheng: Flexible graphene-based lithium ion batteries with ultrafast charge and discharge rates. Proc. Natl. Acad. Sci. U.S.A. 109, 17360-17365 (2012).

11. K.S. Kim, Y. Zhao, H. Jang, S.Y. Lee, J.M. Kim, J.H. Ahn, P. Kim, J.Y. Choi, and B.H. Hong: Large-scale pattern growth of graphene films for stretchable transparent electrodes. Nature 457, 706-710 (2009).

12. X. Meng, X-Q. Yang, and X. Sun: Emerging applications of atomic layer deposition for lithium-ion battery studies. Adv. Mater. 24, 3589-3615 (2012).

13. L. Ci, L. Song, C.H. Jin, D. Jariwala, D.X. Wu, Y.J. Li, A. Srivastava, Z.F. Wang, K. Storr, L. Balicas, F. Liu, and P.M. Ajayan: Atomic layers of hybridized boron nitride and graphene domains. Nat. Mater. 9, 430-435 (2010).

14. K.P. Loh, Q.L. Bao, G. Eda, and M. Chhowalla: Graphene oxide as a chemically tunable platform for optical applications. Nat. Chem. 2, 1015-1024 (2010). 
15. C. Lee, X.D. Wei, J.W. Kysar, and J. Hone: Measurement of the elastic properties and intrinsic strength of monolayer graphene. Science 321, 385-388 (2008).

16. K.S. Novoselov, A.K. Geim, S.V. Morozov, D. Jiang, Y. Zhang, S.V. Dubonos, I.V. Grigorieva, and A.A. Firsov: Electric field effect in atomically thin carbon films. Science 306, 666-669 (2004).

17. P. Sutter: Epitaxial graphene: How silicon leaves the scene. Nat. Mater. 8, 171-172 (2009).

18. L. Tao, J. Lee, M. Holt, H. Chou, S.J. McDonnell, D.A. Ferrer, M.G. Babenco, R.M. Wallace, S.K. Banerjee, R.S. Ruoff, and D. Akinwande: Uniform wafer-scale chemical vapor deposition of graphene on evaporated $\mathrm{Cu}$ (111) film with quality comparable to exfoliated monolayer. J. Phys. Chem. C 116, 24068-24074 (2012).

19. S. Park and R.S. Ruoff: Chemical methods for the production of graphenes. Nat. Nanotechnol. 4, 217-224 (2009).

20. A.K. Geim: Graphene: Status and prospects. Science 324, 15301534 (2009).

21. S.Y. Zhou, G.H. Gweon, A.V. Fedorov, P.N. First, W.A. de Heer, D.H. Lee, F. Guinea, A.H. Castro Neto, and A. Lanzara: Substrateinduced bandgap opening in epitaxial graphene. Nat. Mater. 6, 770-775 (2007)

22. H.J. Park, J. Meyer, S. Roth, and V. Skákalová: Growth and properties of few-layer graphene prepared by chemical vapor deposition. Carbon 48, 1088-1094 (2010).

23. G-H. Lee, R.C. Cooper, S.J. An, S. Lee, A. van der Zande, N. Petrone, A.G. Hammerberg, C. Lee, B. Crawford, W. Oliver, J.W. Kysar, and J. Hone: High-strength chemical-vapordeposited graphene and grain boundaries. Science $\mathbf{3 4 0}$, 1073-1076 (2013).

24. D. Wei, Y. Liu, Y. Wang, H. Zhang, L. Huang, and G. Yu: Synthesis of n-doped graphene by chemical vapor deposition and its electrical properties. Nano Lett. 9, 1752-1758 (2009).

25. W. Cai, R.D. Piner, F.J. Stadermann, S. Park, M.A. Shaibat, Y. Ishii, D. Yang, A. Velamakanni, J.A. Sung, M. Stoller, J. An, D. Chen, and R.S. Ruoff: Synthesis and solid-state NMR structural characterization of 13C-labeled graphite oxide. Science 321, 1815-1817 (2008).

26. Y.W. Zhu, S. Murali, W.W. Cai, X.S. Li, J.W. Suk, J.R. Potts, and R.S. Ruoff: Graphene and graphene oxide: Synthesis, properties, and applications. Adv. Mater. 22, 3906 (2010).

27. K.S. Novoselov, D. Jiang, F. Schedin, T.J. Booth, V.V. Khotkevich, S.V. Morozov, and A.K. Geim: Twodimensional atomic crystals. Proc. Natl. Acad. Sci. U.S.A. 102, 10451-10453 (2005).

28. J.N. Coleman, M. Lotya, A. O'Neill, S.D. Bergin, P.J. King, U. Khan, K. Young, A. Gaucher, S. De, R.J. Smith, I.V. Shvets, S.K. Arora, G. Stanton, H-Y. Kim, K. Lee, G.T. Kim, G.S. Duesberg, T. Hallam, J.J. Boland, J.J. Wang, J.F. Donegan, J.C. Grunlan, G. Moriarty, A. Shmeliov, R.J. Nicholls, J.M. Perkins, E.M. Grieveson, K. Theuwissen, D.W. McComb, P.D. Nellist, and V. Nicolosi: Two-dimensional nanosheets produced by liquid exfoliation of layered materials. Science 331, 568571 (2011)

29. D. Golberg, Y. Bando, Y. Huang, T. Terao, M. Mitome, C. Tang, and C. Zhi: Boron nitride nanotubes and nanosheets. ACS Nano 4, 2979-2993 (2010).

30. Y. Gao, W. Ren, T. Ma, Z. Liu, Y. Zhang, W-B. Liu, L-P. Ma, $\mathrm{X}$. Ma, and H-M. Cheng: Repeated and controlled growth of monolayer, bilayer and few-layer hexagonal boron nitride on $\mathrm{Pt}$ foils. ACS Nano 7, 5199-5206 (2013).

31. C. Gómez-Navarro, M. Burghard, and K. Kern: Elastic properties of chemically derived single graphene sheets. Nano Lett. 8, 20452049 (2008).
32. J.W. Suk, R.D. Piner, J. An, and R.S. Ruoff: Mechanical properties of monolayer graphene oxide. ACS Nano 4, 65576564 (2010)

33. H.D. Espinosa, B.C. Prorok, and B. Peng: Plasticity size effects in free-standing submicron polycrystalline FCC films subjected to pure tension. J. Mech. Phys. Solids 52, 667-689 (2004).

34. P.Y. Huang, C.S. Ruiz-Vargas, A.M. van der Zande, W.S. Whitney, M.P. Levendorf, J.W. Kevek, S. Garg, J.S. Alden, C.J. Hustedt, Y. Zhu, J. Park, P.L. McEuen, and D.A. Muller: Grains and grain boundaries in single-layer graphene atomic patchwork quilts. Nature 469, 389-392 (2011).

35. C.S. Ruiz-Vargas, H.L.L. Zhuang, P.Y. Huang, A.M. van der Zande, S. Garg, P.L. McEuen, D.A. Muller, R.G. Hennig, and J. Park: Softened elastic response and unzipping in chemical vapor deposition graphene membranes. Nano Lett. 11, 2259-2263 (2011).

36. Ç.Ö. Girit, J.C. Meyer, R. Erni, M.D. Rossell, C. Kisielowski, L. Yang, C.H. Park, M.F. Crommie, M.L. Cohen, S.G. Louie, and A. Zettl: Graphene at the edge: Stability and dynamics. Science 323, 1705-1708 (2009).

37. S.A. Joyce and J.E. Houston: A new force sensor incorporating force-feedback control for interfacial force microscopy. Rev. Sci. Instr. 62, 710-715 (1991)

38. D.A. Dikin, S. Stankovich, E.J. Zimney, R.D. Piner, G.H.B. Dommett, G. Evmenenko, S.T. Nguyen, and R.S. Ruoff: Preparation and characterization of graphene oxide paper. Nature 448, 457-460 (2007).

39. L. Song, L. Ci, H. Lu, P.B. Sorokin, C. Jin, J. Ni A.G. Kvashnin, D.G. Kvashnin, J. Lou, B.I. Yakobson, and P.M. Ajayan: Large scale growth and characterization of atomic hexagonal boron nitride layers. Nano Lett. 10, 3209-3215 (2010).

40. A. Castellanos-Gomez, M. Poot, G.A. Steele, H.S.J. van der Zant, N. Agrait, and G. Rubio-Bollinger: Elastic properties of freely suspended $\mathrm{MoS}_{2}$ nanosheets. Adv. Mater. 24, 772 (2012).

41. T. Filleter, J.L. McChesney, A. Bostwick, E. Rotenberg, K.V. Emtsev, T. Seyller, K. Horn, and R. Bennewitz: Friction and dissipation in epitaxial graphene films. Phys. Rev. Lett. 102, 086102 (2009)

42. C. Lee, Q.Y. Li, W. Kalb, X.Z. Liu, H. Berger, R.W. Carpick, and J. Hone: Frictional characteristics of atomically thin sheets. Science 328, 76-80 (2010).

43. J. Ou, J. Wang, S. Liu, B. Mu, J. Ren, H. Wang, and S. Yang: Tribology study of reduced graphene oxide sheets on silicon substrate synthesized via covalent assembly. Langmuir 26, 15830-15836 (2010).

44. Z.Q. Wei, D.B. Wang, S. Kim, S.Y. Kim, Y.K. Hu, M.K. Yakes, A.R. Laracuente, Z.T. Dai, S.R. Marder, C. Berger, W.P. King, W.A. de Heer, P.E. Sheehan, and E. Riedo: Nanoscale tunable reduction of graphene oxide for graphene electronics. Science 328, 1373-1376 (2010).

45. K. Kim, V.I. Artyukhov, W. Regan, Y.Y. Liu, M.F. Crommie, B.I. Yakobson, and A. Zettl: Ripping graphene: Preferred directions. Nano Lett. 12, 293-297 (2012).

46. T. Zhang, X.Y. Li, S. Kadkhodaei, and H.J. Gao: Flaw insensitive fracture in nanocrystalline graphene. Nano Lett. 12, 4605-4610 (2012).

47. J.S. Kim, T. LaGrange, B.W. Reed, M.L. Taheri, M.R. Armstrong, W.E. King, N.D. Browning, and G.H. Campbell: Imaging of transient structures using nanosecond in situ TEM. Science 321, 1472-1475 (2008).

48. Z. Yong, L. Xinyu, R. Changhai, Z. Yan Liang, D. Lixin, and S. Yu: Piezoresistivity characterization of synthetic silicon nanowires 
using a MEMS device. J. Microelectromech. Syst. 20, 959-967 (2011).

49. J. Li, Y. Zhang, S. To, L. You, and Y. Sun: Effect of nanowire number, diameter, and doping density on Nano-FET biosensor sensitivity. ACS Nano 2011, 5, 6661-6668 (2011).

50. H.D. Espinosa, R.A. Bernal, and T. Filleter: In situ TEM electromechanical testing of nanowires and nanotubes. Small 8, 3233 3252 (2012).
51. K.H. Lee, H-J. Shin, J. Lee, I-Y. Lee, G-H. Kim, J-Y. Choi, and S-W. Kim: Large-scale synthesis of high-quality hexagonal boron nitride nanosheets for large-area graphene electronics. Nano Lett. 12, 714-718 (2012).

52. L. Nikolova, T. LaGrange, B.W. Reed, M.J. Stern, N.D. Browning, G.H. Campbell, J.C. Kieffer, B.J. Siwick, and F. Rosei: Nanocrystallization of amorphous germanium films observed with nanosecond temporal resolution. Appl. Phys. Lett. 97, 203102-203103 (2010). 